\title{
CYCLE BIOLOGIQUE DE DRUSUS RECTUS (TRICHOPTERA) \\ DANS LES PYRENÉES CENTRALES : INFLUENCE DE LA TEMPÉRATURE ET DE L'ENNEIGEMENT
}

\author{
par P. Lavandier ${ }^{1}$ et J.-Y. PuJol ${ }^{1}$.
}

La durée du cycle vital de Drusus rectus s'allonge suivant la séquence : biotope déneigé en basse altitude $(1$ an) - biotope déneigé en haute altitude - biotope enneigé en haute altitude ( 2 ans). Les très basses températures déterminent un léger ralentissement de la croissance. A température égale, ce ralentissement est beaucoup plus accusé sous la neige que dans les biotopes déneigés : il affecte principalement les premiers stades larvaires phytophages, qui ne se développent pratiquement pas en absence de périphyton. La taille des larves et des adultes est fonction de la longueur du cycle biologique.

\section{Life cycle of Drusus rectus (Trichoptera) in the central Pyrenees : influence of temperature and snow-cover.}

The duration of the life cycle of Drusus rectus lengthens with the following sequence of biotopes : biotope without snow at low altitude (1 year) - biotope without snow at high altitude - biotope covered with snow at high altitude (2 years). Very low temperatures cause a slight retardation of growth. At a similar temperature, this retardation is much more marked under the snow than in the biotopes that are without snow, and chiefly affects the first-instar larvae. These are phytophagous and do not develop in the absence of periphyton. The size of both the larvae and the adults is related to the length of the life cycle.

Drusus rectus (Mc Lachlan) est un Trichoptère Limnephilidae de type râcleur de substrat. Trois sous-espèces sont connues (Décamps 1972) : Drusus rectus rectus étudiée ici est propre aux Pyrénées centrales et orientales; elle forme d'abondantes populations dans les cours d'eau de haute et moyenne montagne. Le cycle de développement de Drusus rectus varie avec l'altitude (Décamps 1967) c'est-à-dire principalement selon les conditions de température et d'enneigement. Le choix de localités d'études appropriées nous a permis de préciser les modalités de développement de l'espèce, en dissociant l'action de la température et de l'enneigement sur la croissance larvaire.

1. Laboratoire d'Hydrobiologie, Université Paul-Sabatier, 118, route de Narbonne, 31077 Toulouse Cedex, France. 


\section{STATIONS - MATÉRIEL — MẼTHODES}

Nous avons retenu cinq stations dans le réseau hydrographique de la vallée d'Aure :

- le torrent d'Estaragne vers la source à $2350 \mathrm{~m}$ (station 1) ;

- la source d'une tourbière située à $2190 \mathrm{~m}$ sur le flanc sud du pic d'Anglade (station 2) ;

- le ruisseau de Merlans à $2000 \mathrm{~m}$ (station 3) ;

- le ruisseau d'Estaragne près de son embouchure à $1850 \mathrm{~m}$ (station 4);

- le ruisseau de l'Espiaube à $1290 \mathrm{~m}$ (station 5).

Ces milieux sont décrits de façon détaillée par Berthélemy (1966), Décamps (1967), Lavandier (1974). Les caractéristiques principales des stations et le matériel larvaire récolté sont indiqués dans le tableau ci-après :

Stations

Cours d'eau

Altitude

Enneigement

Températures

extrêmes annuelles

Amplitudes thermiques

journalières maximales

Nombre de larves

récoltées
1

Estaragne Tourbière Merlans Estaragne Espiaube $2350 \mathrm{~m} \quad 2190 \mathrm{~m} \quad 2000 \mathrm{~m} \quad 1850 \mathrm{~m} \quad 1290 \mathrm{~m}$

6-7 mois

2$$
0-4{ }^{\circ} \mathrm{C} \quad 0-3,5{ }^{\circ} \mathrm{C} \quad 0-16{ }^{\circ} \mathrm{C} \quad 0-13{ }^{\circ} \mathrm{C} \quad 1-14{ }^{\circ} \mathrm{C}
$$

$$
2{ }^{\circ} \mathrm{C} \quad 2{ }^{\circ} \mathrm{C} \quad 8^{\circ} \mathrm{C} \quad 6{ }^{\circ} \mathrm{C} \quad 6{ }^{\circ} \mathrm{C}
$$

$\begin{array}{lllll}2346 & 800 & 7325 & 247 & 4731\end{array}$

\section{Remargues}

- A la tourbière (station 2), le débit et la morphométrie de la source ne permettent pas l'installation d'une couverture neigeuse. Malgré l'altitude $(2190 \mathrm{~m})$ le biotope reste constamment déneigé. Ainsi, les stations 1 et 2 présentent des températures comparables mais des durées d'enneigement différentes; les stations 2 et 5 ont des températures différentes et des conditions d'enneigement analogues.

- L'abondance des larves est très variable selon les biotopes : en moyenne, moins de 15 larves au $\mathrm{m}^{2}$ à la station $4 \mathrm{du}$ torrent d'Estaragne, de 200 à 500 individus par $\mathrm{m}^{2}$ aux stations $1,2,5$, plus de 1500 dans le ruisseau de Merlans (station 3). Dans cette localité, Drusus rectus est malgré son abondance largement dominé par une espèce voisine Drusus discolor (Rambur).

Nous avons suivi le développement de Drusus rectus entre septembre 1971 et juillet 1973. Durant la période d'accessibilité des stations, des prélèvements de faune ont été réalisés avec une fréquence variant de 15 jours à 2 mois. 
Les adultes ont été capturés par chasses.

Les larves ont été récoltées à l'aide d'un filet de Surber de vide de maille de $0,15 \mathrm{~mm}, 2,3$ ou 4 relevés étant effectués à chaque échantillonnage. Fixée au formol à $4 \%$, la faune est triée sous la loupe binoculaire, puis conservée dans l'alcool à $75^{\circ}$.

Nous avons déterminé les larves d'après les critères systématiques établis par Décamps et Pujol (1975). Les stades larvaires sont séparés par mesure de la largeur des capsules céphaliques (fig. 1). Lorsque le cycle dure deux ans, un stade donné peut être commun à deux générations : les larves de la cohorte la plus âgée présentent alors un abdomen nettement plus volumineux que celles de la cohorte la plus jeune.

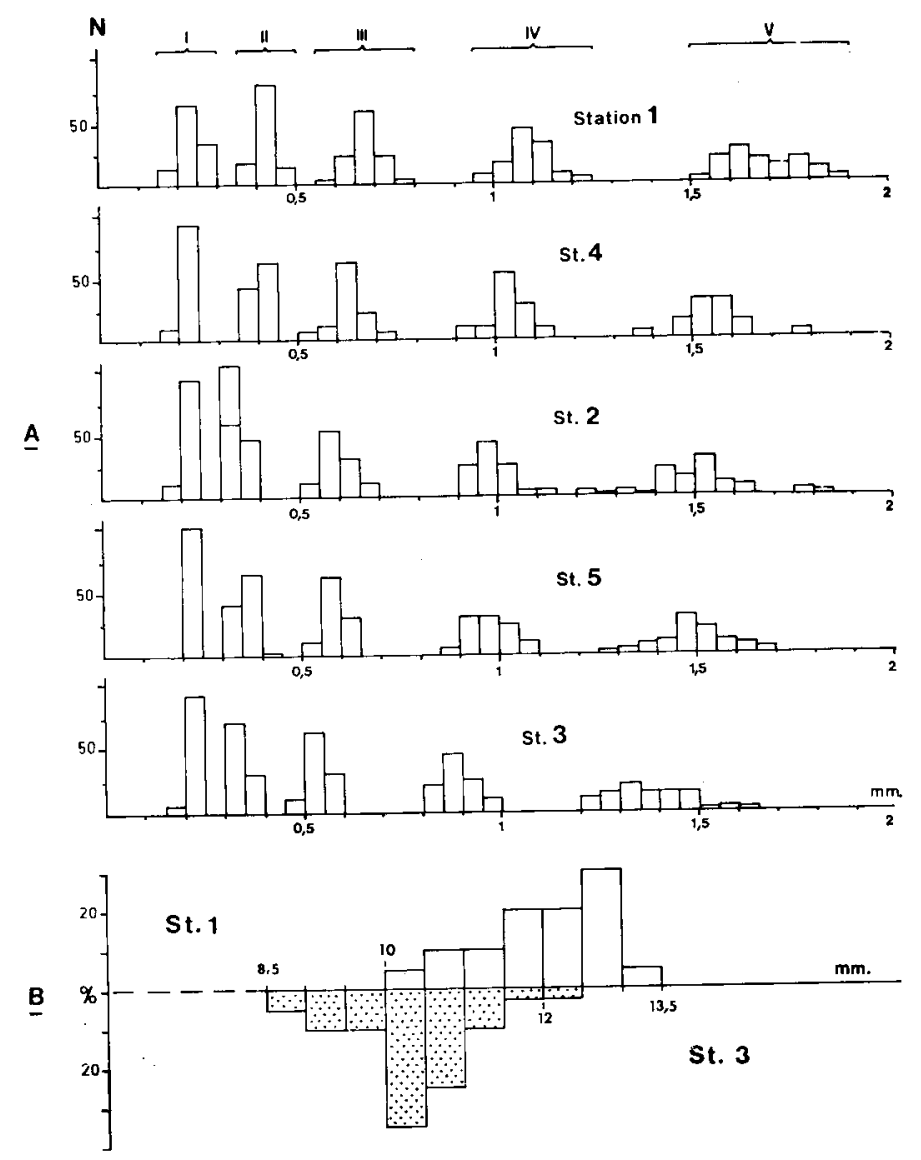

Fig. 1. - A) Largeur des capsules céphaliques des différents stades larvaires aux stations $1,2,3,4$ et 5 (nombre d'individus ramené à 100 pour chaque aux stations 1, 2, 3, 5 et (nombre des des adultes aux stations 1 et 3 (nombres d'individus exprimés en \%). 
Les biomasses sont obtenues par pesées de larves conservées en alcool, puis séchées superficiellement sur papier filtre à température ambiante.

\section{RÉSULTATS}

\section{Variations de taille.}

La mesure des capsules céphaliques de Drusus rectus révèle des différences de taille parfois importantes entre populations ( fig. 1 a). Elles sont perceptibles dès le deuxième stade et s'accentuent au cours du développement. Les larves les plus grandes peuplent l'Estaragne (station 1), les plus petites le Merlans (station 3). Ces différences de taille se répercutent nettement au niveau des adultes (fig. $1 \mathrm{~b}$ ).

\section{Cycles de développement.}

L'interprétation des cycles biologiques est compliquée par l'absence quasi générale des nymphes dans nos récoltes. Seules trois nymphes ont été recueillies en milieu aquatique (stations 1 et 3 ) ; trois autres ont été trouvées en milieu humide, sous un rooher exondé près des berges (station 1). D'autre part, nous avons capturé deux larves au stade $\mathrm{V}$ sur un tronc d'arbre abattu, à près d'un mètre du torrent (station 4). Ces faits traduisent certainement une migration des larves avant la nymphose, sans doute dans le domaine subaquatique.

— Station 1 : le torrent d'Estaragne à $2350 \mathrm{~m}$ (fig. 2.1).

Les premières éclosions ont lieu en octobre et la majeure partie de la population est au stade I quand le torrent se recouvre de neige. En hiver, la croissance est très faible (fig. 3). Quand le torrent réapparaît en juillet, les stades II (jeunes) dominent. Durant la période déneigée la croissance est plus rapide; en novembre la population est aux stades III, IV, V et nymphal. Elle passe ainsi un deuxième hiver avant d'achever son développement l'été suivant.

- Station 2 : source de la Tourbière à $2190 \mathrm{~m}$ (fig. 2.2).

Les éclosions ont lieu en novembre. En absence de couverture neigeuse la croissance se poursuit durant l'hiver (fig. 3) et les larves aux stades II et III dominent au printemps. Le développement se poursuit régulièrement au cours de l'été et les individus les plus âgés se métamorphosent certainement en automne après une seule année de vie larvaire. Le reste de la population passe un deuxième hiver aux stades IV, $V$ et nymphal. Les premiers adultes apparaissent en juin, mais nous n"avons trouvé les premières pontes qu'en août. 
- Station 3 : le ruisseau de Merlans à $2000 \mathrm{~m}$ (fig. 2.3).

Les éclosions sont étalées de septembre à novembre. La croissance des larvules est très faible quand le ruisseau est recouvert ; elle s'accélère dès la fonte des neiges (mars). La majeure partie de la population, encore au stade I en mars, est au stade II en juin, aux stades IV et $\mathrm{V}$ et nymphal (?) en novembre. Les larves passent alors un deuxième hiver avant d'achever leur croissance l'été suivant. Les adultes apparaissent en août.

\section{Stades}
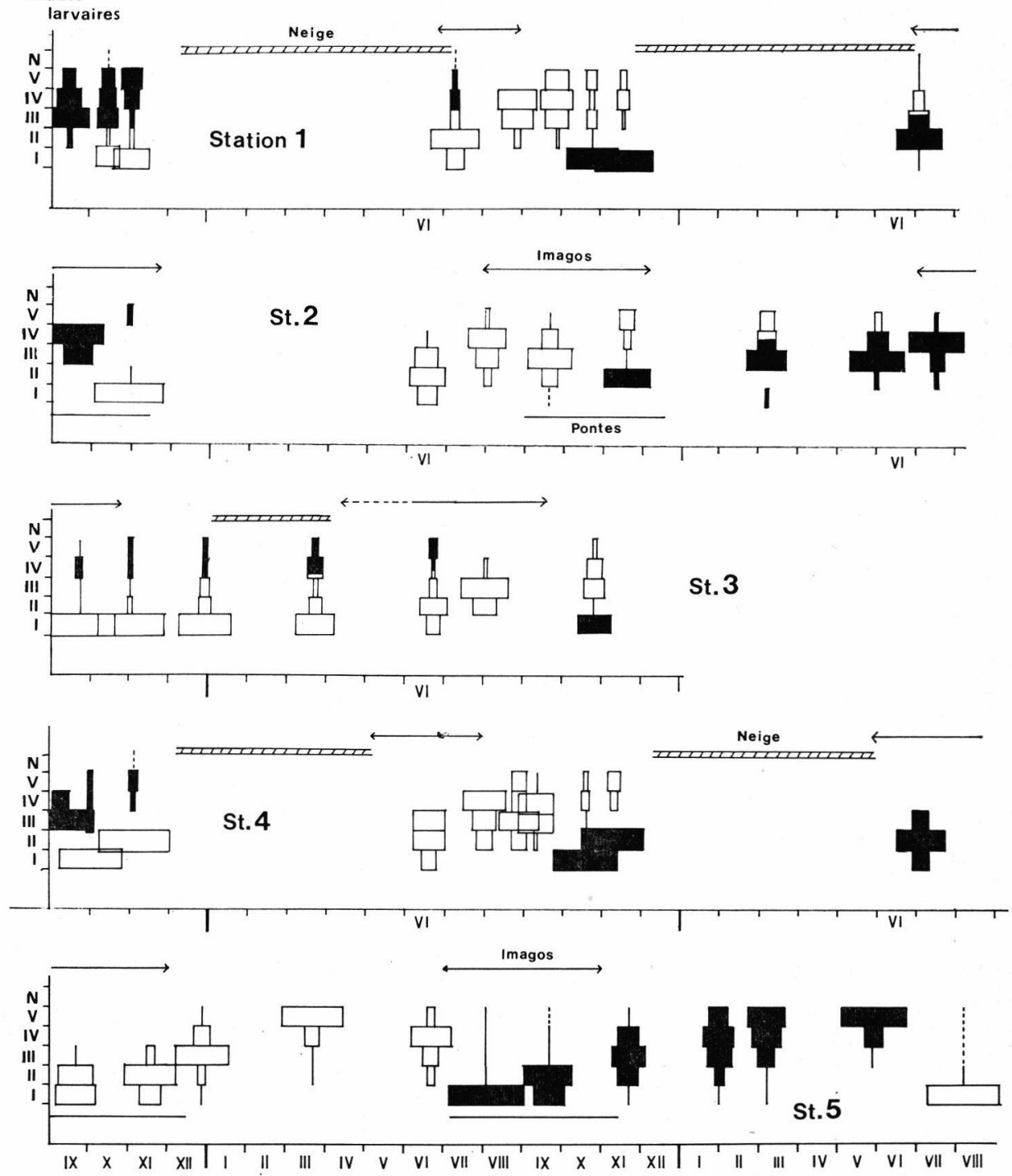

FIg. 2. - Évolution des populations de Drusus rectus aux différentes stations : $\%$ des stades larvaires à chaque date de prélèvement. 
- Station 4 : le torrent d'Estaragne à $1850 \mathrm{~m}$ (fig. 2.4).

Les éclosions ont lieu en septembre et les larves au deuxième stade dominent à la fin de l'automne. La croissance hivernale est très faible (fig. 3) ; en juin les trois premiers stades larvaires coexistent. Le développement s'accélère en été et les premières larves au stade $\mathrm{V}$ apparaissent en août. Ainsi, il est possible qu'après la nymphose une faible partie de la population donne des imagos avant l'hiver (nous n'en avons toutefois jamais capturés après le mois d'août). En novembre, seuls subsistent les derniers stades ; au printemps suivant, on ne trouve plus de larves âgées. Les adultes volent dès le mois de mai.

- Station 5 : le ruisseau d'Espiaube à $1290 \mathrm{~m}$ (fig. 2.5).

Nos résultats corroborent les observations faites par Décamps (1967) dans ce biotope. La période de ponte s'étale de juin à septembre. La plus grande partie de la population est au stade I en juillet, au stade II en août, au stade III en novembre-décembre. La croissance se poursuit durant l'hiver (fig. 3) ; le stade IV domine de janvier à mars, le stade $V$ en mai-juin. Les adultes apparaissent à la fin du mois de juin.

Ainsi, le cycle biologique de Lrusus rectus se déroule selon trois modalités :

- la population passe un hiver avant de se métamorphoser ;

- la population passe deux hivers avant de se métamorphoser ;

- la population présente un développement intermédiaire aux deux types précédents : une partie donne des imagos à l'automne, l'autre durant le printemps et l'été suivant. L'importance relative de ces deux parties peut fluctuer d'une année à l'autre selon les conditions météorologiques locales.

\section{DISCUSSION}

Plusieurs espices de Limnephilidae montrent une diminution de taille liée à l'élévation en altitude (Schmid 1951). Cette diminution de taille, due à la réduction de la période de nutrition, permet à l'espèce d'accomplir son cycle vital aussi rapidement en montagne qu'en plaine, malgré le ralentissement hivernal de la croissance (Décamps 1967). Chez Drušs rectus, la durée de la vie larvaire et la taille des individus augmentent de façon parallèle selon la séquence : biotope déneigé en basse altitude - biotope déneigé en haute altitude - biotope enneigé en haute altitude. Toutefois, la population du Merlans (station 3 ) ne s'intègre pas à ce schéma d'ensemble. L'explication doit en être recherchée dans le régime thermique du cours d'eau (fortes amplitudes quotidiennes des températures) et dans l'importance que prennent à cette station les phénomènes de concurrence intra- ou interspécifiques (avec Drusus discolor). 
L'étude comparée des populations (particulièrement aux stations 1,2 et 5 ) met en évidence le rôle respectif de la nieige et de la température sur le développement.

* En absence de couverture de neige.

- Quelle que soit la température ou la saison, il n'y a pas arrêt de développement (fig. 2 et 3 ). Cependant les très basses températı:es entraînent un ralentissement de la croissance (nettement perceptible dans la comparaison du développement des premiers stades larvaires aux diverses stations ou dans l'évolution de la croissance au cours de l'année).

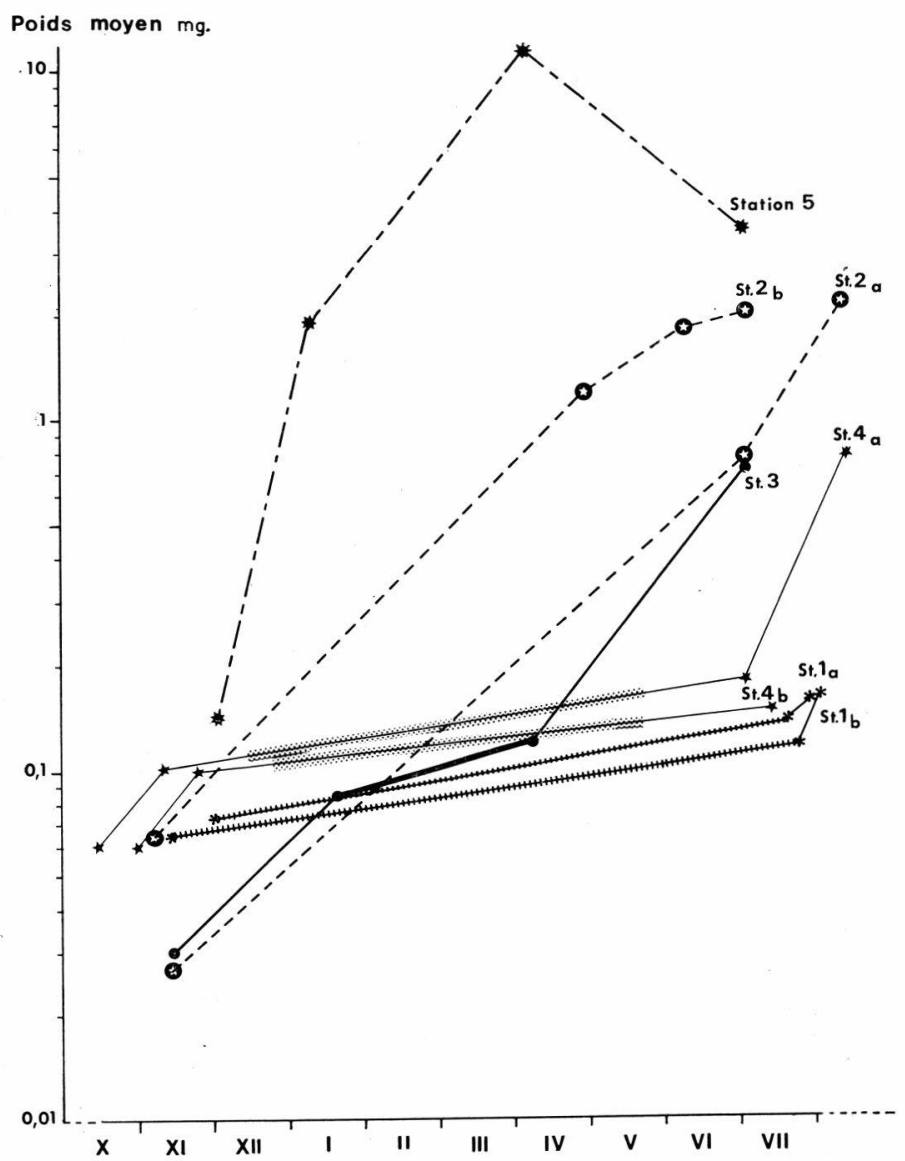

Fig. 3. - Croissance hivernale du poids moyen de Drusus rectus aux stations $1,2,3,4$ et 5 (a : larves écloses en $1971 ; \mathrm{b}:$ larves écloses en 1972). Les figurés correspondent aux périodes d'enneigement des biotopes. 
- Le développement de Drusus rectus est comparable à chaque station. Il paraît toutefois d'autant plus rapide qu'il s'effectue à des températures modérées et en absence de concurrence : jl est maximum au début de l'hiver dans l'Espiaube (station 5); il est plus rapide dans l'Estaragne (station 4) qu'au Merlans (station 3).

* Sous la Neige.

- La croissance est très faible. Dans la figure 3 qui résume les modalités de développement hivernal, il apparaît clairement qu'à température égale, c'est l'enneigement qui détermine les caractéristiques de la croissance. La reprise du développement est fonction de la durée d'enneigement.

- L'installation de la couverture neigeuse provoque l'arrêt des processus photosynthétiques. L'influence de cet écran est donc d'autant plus sensible qu'elle s'exerce sur les jeunes stades larvaires dont le régime alimentaire spécialisé est à base de périphyton. Les larves âgées, plus omnivores, paraissent moins dépendantes de la luminosité. Or en altitude, lorsque les cours d'cau commencent à se recouvrir, la plus grande partie des populations de Drusus rectus est aux stades I et II.

Les basses températures automnales qui ralentissent l'incubation des œufs et retardent les éclosions ont donc indirectement une influence sur le développement hivernal des populations. Ainsi, la durée du cycle biologique de Drusus rectus dépend en grande partie de la croissance des premiers stades larvaires. Celle-ci est fonction des conditions initiales de température d'une part, de la présence ou de l'absence d'une couverture nivale qui conditionne les possibilités trophiques d'autre part. Les larves âgées paraissent moins dépendantes des conditions de milieu.

\section{TRAVAUX GITÉS}

Berthélémy (C.). 1966. - Recherches écologiques et biogéographiques sur les Plécoptères et Coléoptères d'eau courante (Hydraena et Elminthidae) des Pyrénées. Annls Limnol., 2 (2) : 227-458.

DÉcamps (H.). 1967. - Ecologie des Trichoptères de la vallée d'Aure (Hautes-Pyrénées). Annls Limnol., 3 (3) : 399-577.

DÉcamps (H.) . 1972. - Trichoptères nouveaux des Pyrénées. Nouv. Rev. Ent., 2 : 261-265.

Décamps (H.) et Pujol (J. Y.). 1975. - Les larves de Drusinae des Pyrénées (Trichoptères, Limnephilidae). Annls Limnol., 11 (2) : $157-167$.

Iavandier (P.). 1974. - Écologie d'un torrent pyrénéen de haute montagne. (I. Caractéristiques physiques. Annls Limnol., 10 (2) :179-219.

Schmid (F.). 1951. - Notes sur quelques Halesus (Trich. Limnephilidae). Bull. Soc. Vaud. Sc. nat., 65: 63-71. 